\title{
Mécanismes d'interaction d'une pompe et d'un circuit Modèles théoriques
}

\author{
B. Desmet Ecole nationale supérieure d'arts et métiers, Lille \\ F. Tephany EDF - DER, Chatou \\ J.L. Trolle EDF - DER, Saint-Denis
}

Des effets d'interaction machine-circuit sont mis en évidence et l'importance de la résonance hydraulique sur ces effets est étudiée. Il est nécessaire, en vue de la prévision du comportement dynamique d'un ensemble machine-circuit de caractériser la machine en tant que source de fluctuations, ce qui nécessite de s'affranchir des effets d'interaction lors des essais. Les méthodes linéaires d'analyse théorique sont présentées et critiquées. Le flux d'énergie des fluctuations est exprimé et comparé à la notion classique d'intensité acoustique. Les méthodes théoriques présentées sont utilisées en vue de donner une interprétation des cas recensés.

\section{Introduction}

\subsection{Mise en évidence d'effets d'interaction}

Voici quelques exemples de phénomènes de résonance hydraulique tels qu'on a pu les observer sur des installations industrielles ou susciter leur manifestation sur des boucles d'essais :

\subsubsection{Circuit de centrale nucléaire}

Sur un circuit de centrale thermonucléaire schématisé sur la figure 1, comportant 2 pompes en parallèle alimentées par une bâche, on a fait les constatations suivantes: au cours d'essais réalisés par EDF/DTG pour lesquels l'une des 2 pompes fonctionnait à débit partiel, l'autre étant à l'arrêt, on mesure à l'aspiration de la pompe qui fonctionne des fluctuations de pression organisées à 7,25
$\mathrm{Hz}$ d'une amplitude globale crête à crête de 3,5 bar, et à l'aspiration de la pompe à l'arrêt des fluctuations de pression à la même fréquence et d'un niveau beaucoup plus important, de l'ordre de 14 bar (voir figure 2). Par ailleurs, lorsqu'on intervertit le rôle des 2 pompes, et bien que celles-ci soient situées sur des branches de longueurs différentes, on observe le même phénomène et à la même fréquence, c'est-à-dire qu'il y a symétrie du mécanisme instationnaire en dépit de la dissymétrie géométrique et amplification d'une branche sur l'autre responsable d'une dépression importante dans la branche en eau morte. De surcroît, on mesure des vibrations excessives sur la tuyauterie d'alimentation de la pompe $\varnothing 2 \mathrm{PO}(1 \mathrm{~cm}$ crête à crête) à la fréquence de $14,5 \mathrm{~Hz}$ qui correspond justement à la première fréquence propre mécanique du tronçon rempli d'eau mesurée lors d'excitations par choc.

Interaction phenomena between a pump and a piping system. Theoretical models.

Some machine/circuit interaction effects are disclosed and the significance of hydraulic resonance concerning these effects is examined. Considering the anticipation of the dynamic behaviour of a machine/circuit unit, it is essential to characterize the machine in so far as it is the fluctuations source, which requires that it be freed from the interaction effects during these tests. The theoretical analysis linear methods are presented and critically examined. The energy flux of the fluctuations is expressed and compared to the conventional notion of acoustic intensity. The theoretical methods presented are used to provide an interpretation of recorded cases. 


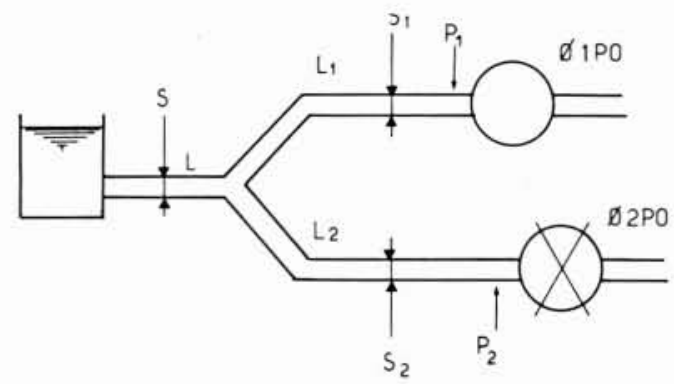

1.

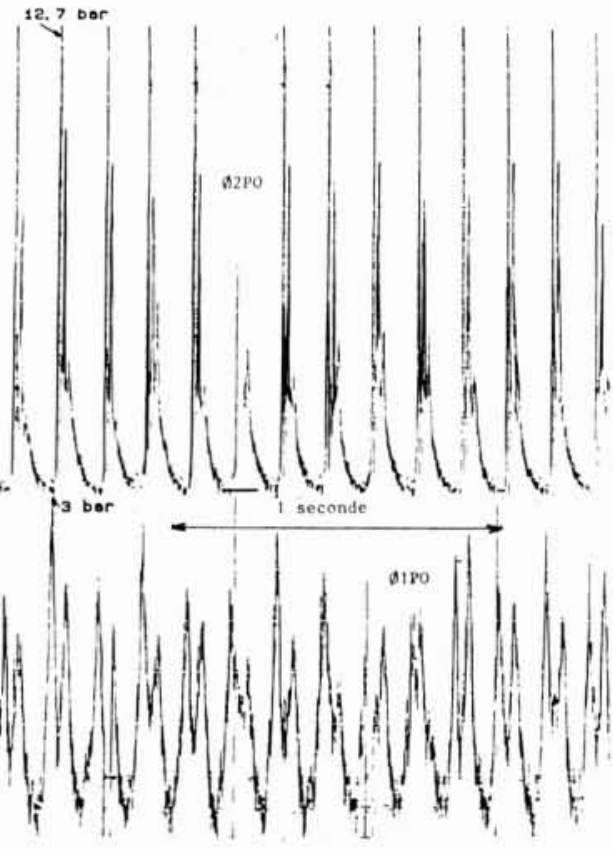

3.

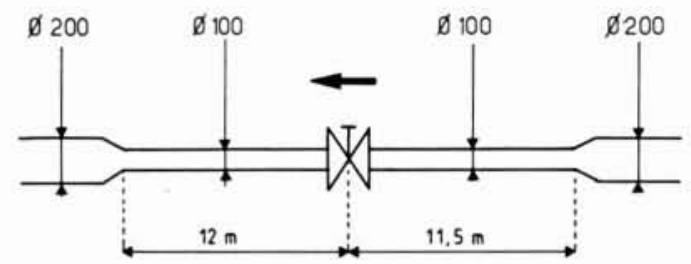

RAPPORT $A(t) / B(f)$ PHASE

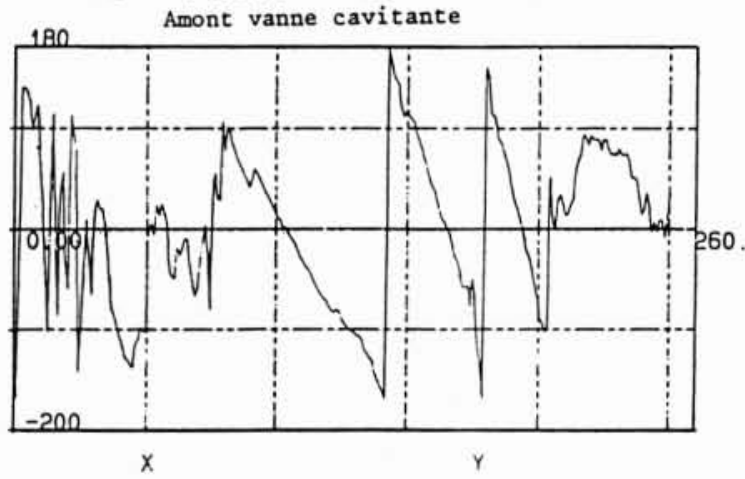

RAPPORT $B(f) / A(f)$ PHASE

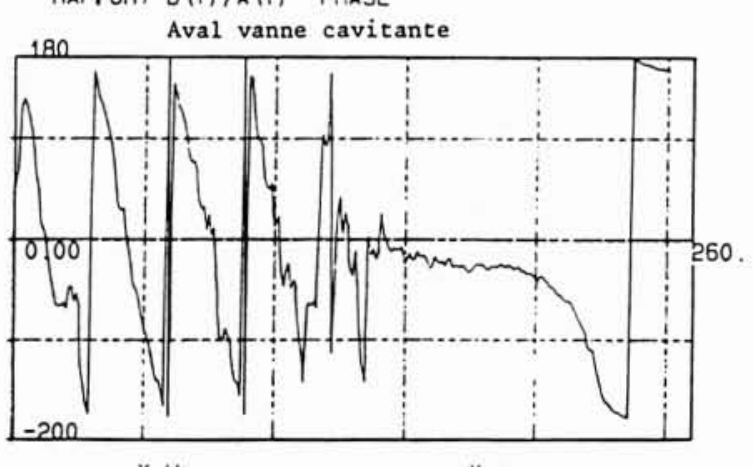

4.

$\mathrm{X} \mathrm{Hz}$

y nor
5.

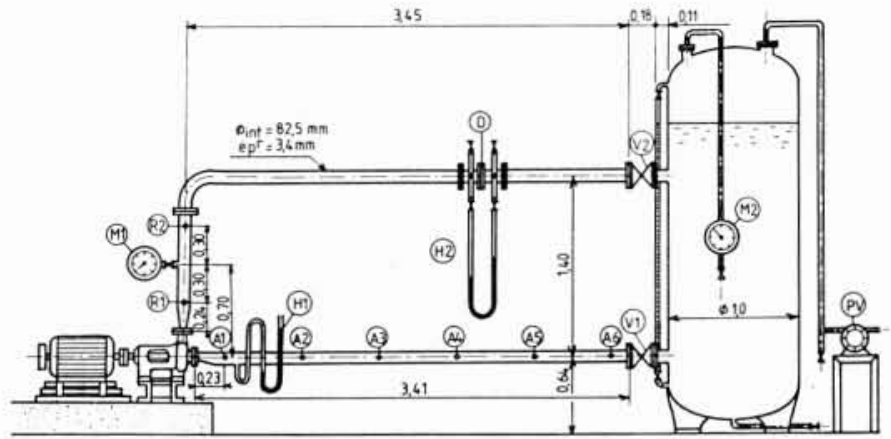

AUTO SPEC CH.A

dB/ 1 bar AMS

6.

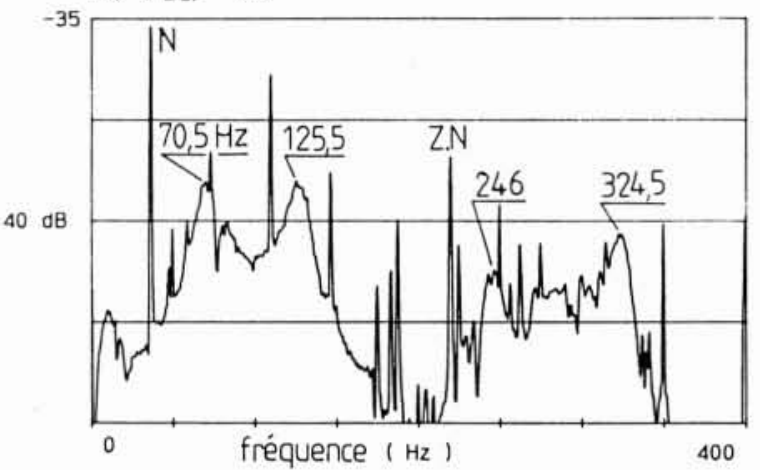

1. Circuit de centrale nucléaire.

2. Fluctuations de pression observées à l'aspiration des pompes.

3. Circuit d'essais de vannes.

4. Célérité des ondes à l'amont et à l'aval de la vanne cavitante.

5. Boucle d'essais de pompes.

6. Analyse en fréquence de la pression à l'aspiration de la pompe (point A1). $N=2200 \mathrm{tr} / \mathrm{min}$.

7. Boucle "SESAME».

7.

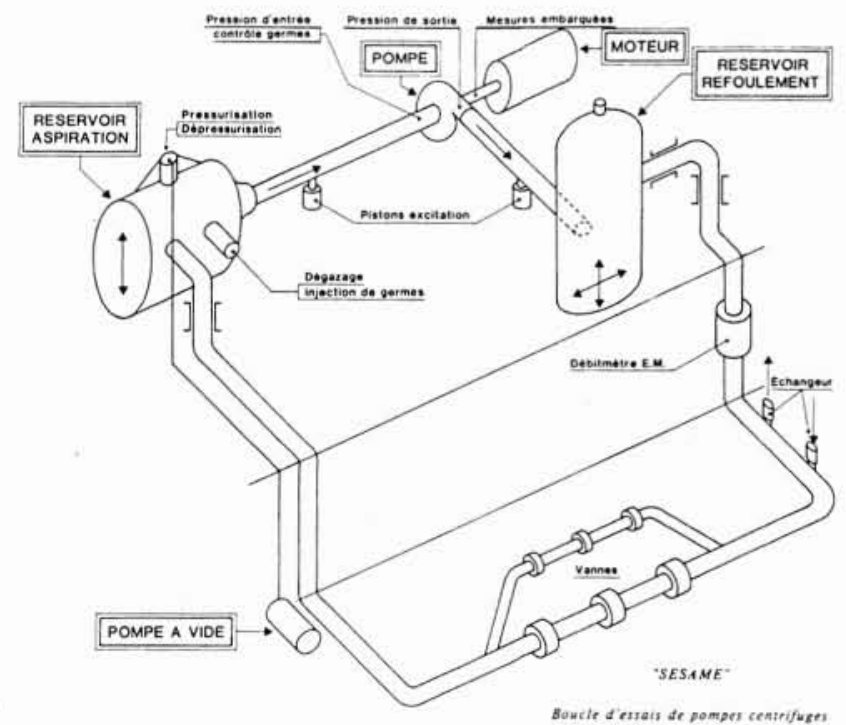




\subsubsection{Essais d'une vanne papillon}

Au cours d'essais réalisés par la DER sur une vanne papillon fonctionnant successivement hors cavitation et en régime fortement cavitant, on a mis en évidence un phénomène de résonance hydraulique portant sur les portions situées en amont et en aval de la vanne jusqu'aux éléments de raccordements de la boucle à une conduite de diamètre supérieur (figure 3 ). La présence de cavitation pour le $2^{\mathrm{e}}$ cas de fonctionnement dans la zone aval se traduit par une vitesse de propagation des ondes beaucoup plus faible qu'en amont (figure 4), faisant apparaître des fréquences naturelles différentes de part et d'autre de la vanne.

\subsubsection{Essais d'une pompe centrifuge}

On a pu exciter les résonances hydrauliques sur une petite boucle d'essais installée à l'ENSAM de Lille ( $f$ gure 5), en faisant varier la vitesse de rotation de la pompe: les différentes harmoniques sont exaltées au passage des fréquences naturelles du circuit, l'allure des fluctuations pouvant être sensiblement modifiée selon l'ordre de l'harmonique amplifié (figure 6 et références [1], [2]).

\subsection{Caractérisation d'une machine en tant que source de fluctuations}

La principale difficulté pour caractériser une machine en tant que source de fluctuations, indépendamment de son circuit d'implantation, est de s'affranchir des effets d'interaction machine-circuit. Deux stratégies peuvent être envisagées pour résoudre ce problème. La première consiste à placer la machine dans un circuit dont on sait modéliser la zone expérimentale (conduites rectilignes) et en isolant la machine des autres sources de fluctuations (réservoirs) comme dans le cas de la boucle "SESAME " de l'ENSAM-Lille (figure 7) par exemple. Le traitement des signaux s'appuie sur le modèle théorique pour restituer l'information utile. Une autre possibilité est de faire disparaître les phénomènes d'ondes quasi-stationnaires par l'emploi de terminaisons anéchoïques.

\section{Modélisation et interprétation}

\subsection{Méthodes d'analyse théorique des phéno- mènes}

\subsubsection{Examen critique des hypothèses classiques de la théorie de propagation en ondes planes}

Le modèle d'ondes planes (réf. [3] à [5]) est souvent utilisé pour l'étude des phénomènes d'interaction machine-circuit. Dans ce modèle, seule la composante de la vitesse suivant l'axe de la conduite est considérée et cette composante, ainsi que les autres caractéristiques de l'écoulement (pression, masse volumique du fluide...) sont supposées uniformes dans toute section droite à chaque instant.

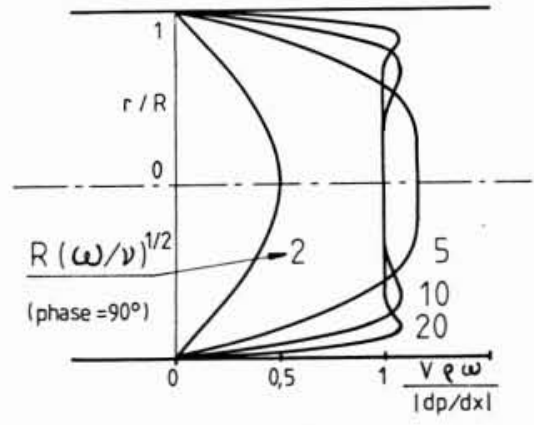

8. Profil des vitesses en fonction du paramètre de fréquence d'après [6].

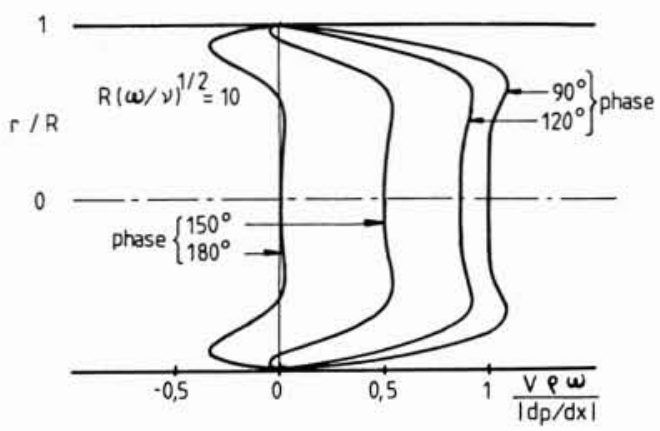

9. Evolution temporelle du profil des vitesses d'après [6].

La figure 8 reproduit les distributions des vitesses axiales $V$ données par Stecki et Davis (réf. [6]) pour un écoulement de fluide visqueux incompressible quand le gradient de pression $d p / d x$ varie dans le temps suivant une loi sinusoïdale de pulsation $\omega$. Le profil des vitesses est caractérisé par un paramètre de fréquence $R(\omega / v)^{1 / 2}$ analogue à un nombre de Reynolds. Pour les valeurs assez grandes de ce paramètre, une zone annulaire où la vitesse est maximale apparait (effet Richardson). La figure 9 montre les évolutions de la vitesse en fonction du temps pour une valeur du paramètre de fréquence égale à 10 .

Le modèle unidimensionnel ne permet pas de tenir compte du profil instationnaire des vitesses. En hydraulique, le paramètre de fréquence est généralement grand $\left(R(\omega / v)^{1 / 2}=250\right.$ pour l'eau pour une fréquence égale à $1 \mathrm{~Hz}$ dans une conduite de diamètre $100 \mathrm{~mm}$ ) et on peut prévoir un profil assez plat des vitesses sauf au voisinage immédiat de la paroi.

Fanelli (réf. [7]) étudie les composantes axiales $V_{X}$ et radiale $V_{r}$ de la vitesse pour une oscillation stationnaire de pulsation $\omega$ dans une conduite cylindrique infinie de section circulaire. Le caractère bidimensionnel des phénomènes apparaît nettement sur la figure 10 (page suiv.) qui représente qualitativement les trajectoires du fluide. Les nœuds de la vitesse longitudinale $\left(V_{X}-0\right)$ coïncident avec les ventres de la vitesse radiale $V_{r}$ et de la pression $p$. 
10.

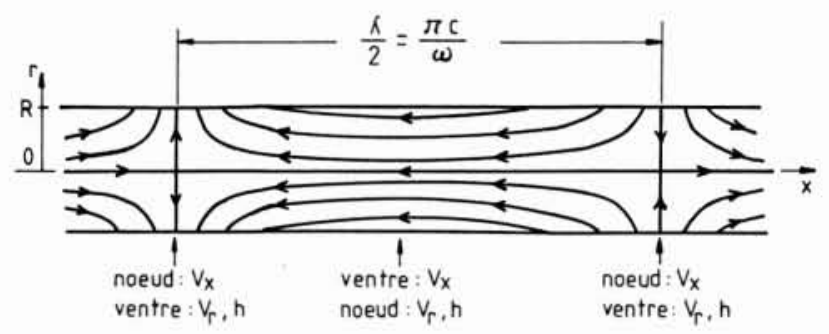

11.

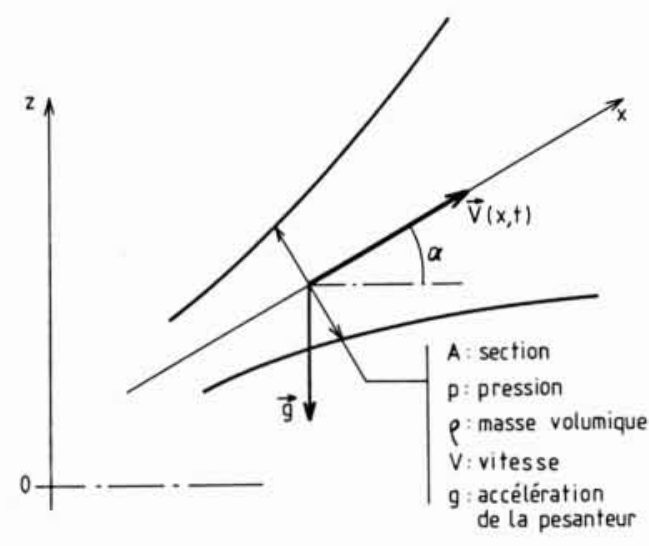

10. Trajectoires du fluide pour une oscillation stationnaire d'après [7].

11. Ecoulement par tranches planes (notations).

12. Fonction de transfert section / pression.

13. Etude énergétique (notations).

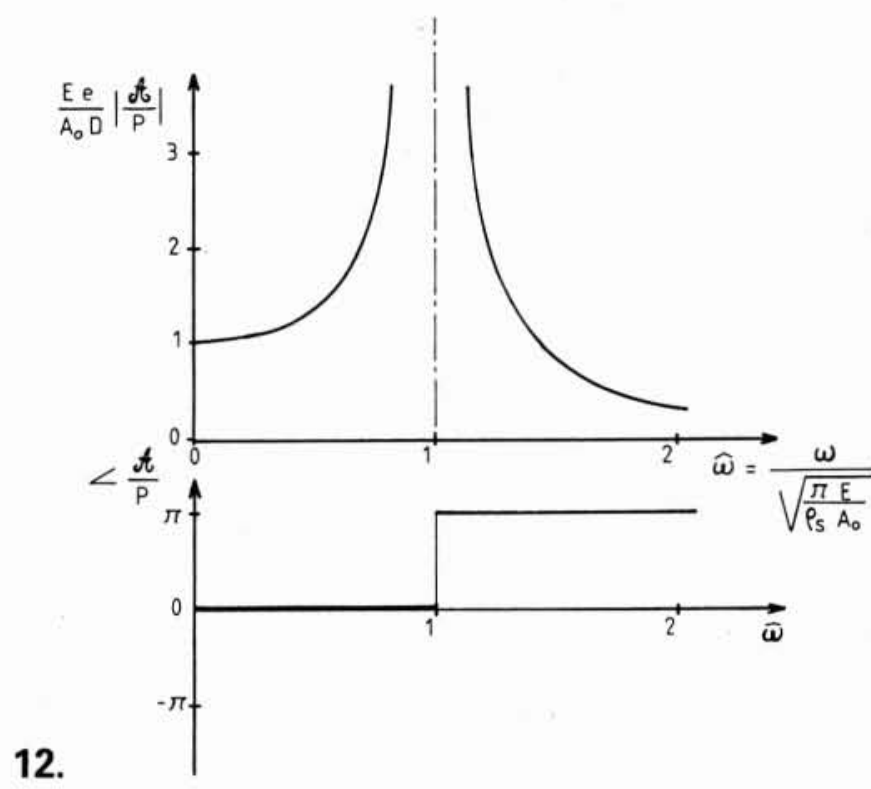

12.

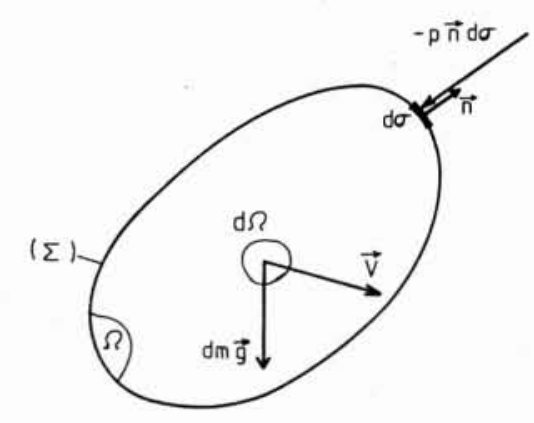

Dans le cadre de l'hypothèse des ondes planes, les équations de continuité et de quantité de mouvement s'écrivent respectivement (figure 11):

$$
\begin{gathered}
\frac{\partial(\rho A)}{\partial t}+\frac{\partial(\rho A V)}{\partial x}=0 \\
\frac{1}{\rho} \frac{\partial p}{\partial x}+\frac{\partial V}{\partial t}+V \frac{\partial V}{\partial x}+g \sin \alpha+\frac{\lambda}{D} \frac{|V| V}{2}=0
\end{gathered}
$$

où le terme de frottement $(\lambda / D)(V|V| / 2)$ est exprimé comme en écoulement permanent.

Les effets capacitifs sont dus à la compressibilité $\chi$ du fluide et au comportement dynamique de la conduite.

$$
\chi=\frac{1}{\rho} \frac{d \rho}{d p}
$$

Dans l'équation de continuité (1) la section $A$ doit être considérée comme fonction de l'abscisse $x$ et du temps $t(A=A(x, t))$. En pratique, on ne tient compte que du comportement statique local lorsqu'on admet la relation de proportionnalité entre la fluctuation de pression dans une tranche et la variation de section. Avec cette dernière hypothèse, la section ne dépend du temps $t$ que par l'intermédiaire de la pression :

$$
A=A(x, p) \quad \text { avec } \quad p=p(x, t)
$$

La figure 12 montre la fonction de transfert sectionpression d'une conduite cylindrique mince présentant une fluctuation de rayon uniforme sur toute sa longueur. Les écarts par rapport au comportement statique apparaissent pour une pulsation nettement inférieure à la pulsation propre (pulsation réduite $\hat{\omega}=1$ ). La fréquence naturelle pour une conduite en acier de $250 \mathrm{~mm}$ de diamètre est égale à $6,6 \mathrm{kHz}$ et les phénomènes dynamiques deviennent sensibles au tiers de cette fréquence environ. En pratique, d'autres modes de déformation peuvent intervenir et des effets de dynamique de structure sont observés pour des fréquences plus basses.

En introduisant la compressibilité $\chi$ et en posant :

$$
c^{2}=\frac{\frac{1}{\chi \rho}}{1+\frac{1}{\chi A} \frac{\partial A(x, p)}{\partial p}}
$$


l'équation de continuité (1) devient :

$\frac{\partial p}{\partial t}+V \frac{\partial p}{\partial x}+\rho c^{2} \frac{\partial V}{\partial x}+\frac{\rho c^{2}}{A} V \frac{\partial A(x, p)}{\partial x}=0$

La quantité $c$ définie par la relation (5) peut être identifiée à la célérité des ondes dans la conduite. La dérivée $\partial A(x, p) / \partial p$ dépend des conditions de liaison de la conduite. Dans le cas particulier des conduites minces, on aboutit à la formule :

$$
c^{2}=\frac{\frac{1}{\chi \rho}}{1+\frac{1}{\chi E} \frac{D}{e} C_{1}}
$$

où $C_{1}=1$ pour une conduite libre sans reprise d'effet de fond (état plan de contraintes dans le plan perpendiculaire à l'axe de la conduite).

$C_{1}=1-v / 2$ pour une conduite libre avec reprise d'effet de fond (état de contraintes pseudo plan)

$C_{1}=1-v$ pour une conduite immobilisée sur toute sa longueur (état plan de déformations).

$E$ représente le module d'élasticité longitudinal et $v$ le coefficient de Poisson du matériau constituant la paroi d'épaisseur $e$.

En hydraulique, la pression est le plus souvent exprimée en hauteur de fluide $(h=p / \rho g$ ).

Les équations de l'écoulement sont transformées en considérant chaque variable comme la somme d'une valeur moyenne (indice 0 ) et d'une quantité fluctuante

$$
h=h_{0}+\tilde{h} \quad V=V_{0}+\tilde{V}
$$

où on considère que les valeurs fluctuantes sont faibles devant les grandeurs moyennes. Enfin, en admettant des variations spatiales lentes par rapport aux variations temporelles, il vient en introduisant le débit volume $q_{v}$ :

$$
\frac{g A_{0}}{c_{0}^{2}} \cdot \frac{\partial \tilde{h}}{\partial t}+\frac{\partial \widetilde{q}_{v}}{\partial x}=0
$$

$\frac{\partial \tilde{h}}{\partial x}+\frac{1}{g A_{0}} \frac{\partial \tilde{q}_{v}}{\partial t}$

$$
+\left(\frac{\lambda}{D}-\frac{1}{A_{0}} \frac{d A_{0}}{d x}\right) \frac{q_{v 0}}{g A_{0}^{2}} \tilde{q}_{v}=0
$$

La suppression des termes $V_{0} \partial \tilde{h} / \partial x$ et $V_{0} \partial \tilde{q}_{v} / \partial x$ respectivement devant $\partial \hat{h} / \partial t$ et $\partial \widetilde{q}_{v} / \partial t$ est convenable si le nombre de Mach de l'écoulement moyen reste petit ce qui est le cas pour les applications en hydraulique sauf, peut-être, en présence de cavitation parce que la célérité $c$ peut devenir faible.

En l'absence d'écoulement moyen, les équations précédentes restent convenables à l'exception du terme de frottement car, dans ce cas, les vitesses s'inversent et la linéarisation effectuée n'est plus valable.

Le terme - $\left(1 / A_{0}\right)\left(d A_{0} / d x\right)\left(q_{v 0} / g A_{0}^{2}\right) \tilde{q}_{v}$ de l'équation (9), dû à la variation de la vitesse moyenne résultant de l'évolution de section joue le rôle d'un frottement négatif dans le cas d'une conduite divergente et ce terme est prépondérant devant la quantité $(\lambda / D)\left(q_{v 0} / g A_{0}^{2}\right) \widetilde{q}_{v}$ dûe au frottement.

\subsubsection{Analyse énergétique des fluctuations de pres- sion}

La caractérisation du bruit aérien des installations par intensimétrie acoustique s'est largement développée au cours de ces dernières années. Plusieurs définitions de l'intensité acoustique, en présence d'écoulement, ont été proposées (réf. [8] à [11]) mais il existe peu de références dans le domaine de l'hydraulique (réf. [12]).

L'exergie $E x$ et l'anergie $A n$ d'un volume quelconque $(\Omega)$ pris dans un milieu fluide en écoulement (figure 13) représentent respectivement la part d'énergie du système qui peut être restituée sous forme d'énergie mécanique quand on ramène ce système jusqu'à un état de référence et la part de cette énergie qui ne peut plus être restituée sous forme d'énergie mécanique.

$$
E x=\int_{\Omega} \rho \text { ex } d \Omega \quad A n=\int_{\Omega} \rho \text { an } d \Omega
$$

Les quantités massiques $e x$ et an sont définies par

$$
\begin{aligned}
& e x=u-u_{a}-T_{a}\left(s-s_{a}\right)+\frac{V^{2}}{2} \\
& a n=T_{a}\left(s-s_{a}\right)
\end{aligned}
$$

où l'indice $a$ se rapporte à l'état de référence.

( $u$ : énergie interne massique, $s$ : entropie massique, $T$ : température)

Par application du premier principe de la thermody. namique au volume $(\Omega)$ :

$$
\begin{aligned}
& \int_{\Omega}\left[\frac{\partial \rho(e x+a n)}{\partial t}-\rho(\vec{g} \cdot \vec{V})\right] d \Omega \\
& \quad+\int_{\Sigma}[\rho(e x+a n)+p](\vec{v} \cdot \vec{n}) d \sigma=0
\end{aligned}
$$

Pour des évolutions isentropiques, l'énergie massique reste constante et, compte tenu de l'équation de continuité, la relation (10) se réduit à :

$$
\begin{aligned}
\int_{\Omega}\left[\frac{\partial(\rho e x)}{\partial t}-\right. & \rho(\vec{g} \cdot \vec{V})] d \Omega \\
& +\int_{\Sigma}(\rho e x+p)(\vec{v} \cdot \vec{n}) d \sigma=0
\end{aligned}
$$

L'intégrale de surface de la relation (11) définit le flux d'énergie à travers la surface $(\Sigma)$ limitant le volume $(\Omega)$. Pour la section droite d'un écoulement unidimensionnel, le flux d'énergie par unité de surface $E$ s'exprime sous la forme :

$$
E=(\rho e x+p) V=E_{p}+E_{c}
$$

avec

$$
\begin{gathered}
E_{p}=\rho V\left[u-u_{a}-T_{a}\left(s-s_{a}\right)\right]+p V \\
E_{c}=\rho \frac{V^{3}}{2}
\end{gathered}
$$

Pour des fluctuations $\tilde{p}$ et $\tilde{V}$ de la pression et de la vitesse autour des conditions moyennes (indice 0 ), en prenant des développements limités du deuxième ordre, les expressions (13) et (14) s'écrivent : 


$$
\begin{aligned}
& E_{p}=\rho_{0} V_{0} e x_{0}+p_{0} V_{0} \\
& +\frac{V_{0} e x_{0}}{c_{0}^{2}} \tilde{p}+\rho_{0} e x_{0} \tilde{V}+\frac{p_{0} V_{0}}{\rho_{0} c_{0}^{2}} \tilde{p} \\
& +p_{0} \tilde{V}+V_{0} \tilde{p}+\tilde{p} \tilde{V}+\frac{e x_{0}}{c_{0}^{2}} \tilde{p} \tilde{V} \\
& +\frac{p_{0}}{\rho_{0} c_{0}^{2}} \tilde{p} V^{\sim}+\frac{p_{0} V_{0}}{\rho_{0}^{2} c_{0}^{2}} \tilde{p}^{2} \\
& +\frac{V_{0} e x_{0}}{2 \rho_{0} c_{0}^{4}} \tilde{p}^{2}+\frac{V_{0}}{2 \rho_{0} c_{0}^{2}}\left(1-\frac{p_{0}}{\rho_{0} c^{2}}\right) \tilde{p}^{2} \\
& E_{c}=\frac{1}{2} \rho_{0} V_{0}^{3}+\frac{V_{0}^{3}}{2 c_{0}^{2}} \tilde{p} \\
& +\frac{3}{2} \rho_{0} V_{0}^{2} \tilde{V}+\frac{3}{2} \frac{V_{0}^{2}}{c_{0}^{2}} \tilde{p} \tilde{V} \\
& +\frac{1}{4} \frac{V_{0}^{3}}{\rho_{0} c_{0}^{4}} \tilde{p}^{2}+\frac{3}{2} \rho_{0} V_{0} \tilde{V}^{2}
\end{aligned}
$$

Les quantités $E_{p_{0}}=\rho_{0} V_{0} e x_{0}+p_{0} V_{0}$ et $E_{c 0}=$ $\rho_{0} V_{0}^{3} / 2$ correspondent au flux d'énergie de l'écoulement moyen et les termes du premier ordre ont une valeur moyenne nulle. Le flux d'énergie moyen $\widetilde{E}$ dû aux fluctuations est donc égal à la valeur moyenne des termes du second ordre :

$$
\begin{aligned}
\overline{\tilde{E}}_{p}=\overline{\tilde{p} \tilde{V}}\left(1+\frac{e x_{0}}{c_{0}^{2}}\right. & \left.+\frac{p_{0}}{\rho_{0} c_{0}^{2}}\right) \\
& +\frac{\overline{\tilde{p}^{2}}}{\rho_{0} c_{0}} \frac{M}{2}\left(1+\frac{e x_{0}}{c_{0}^{2}}+\frac{p_{0}}{\rho_{0} c^{2}}\right) \\
\overline{\widetilde{E}}_{c}=\frac{3}{2} M^{2} \overline{\tilde{p} \tilde{V}} & +\frac{1}{4} M^{3} \frac{\overline{\tilde{p}^{2}}}{\rho_{0} c_{0}}+\frac{3}{2} \rho_{0} V_{0} \overline{\tilde{V}^{2}}
\end{aligned}
$$

Une évaluation numérique des termes des relations (17) et (18) montre que le flux d'énergie par unité de surface moyen peut être confondu avec l'intensité $I$ :

$$
\overline{\widetilde{E}}=\overline{\widetilde{E}}_{p}+\overline{\widetilde{E}}_{c} \simeq \overline{\tilde{p} \tilde{V}}=I
$$

L'intensité $I$ étant un flux d'énergie par unité de surface s'exprime en $W / \mathrm{m}^{2}$ dans le système international.

\subsubsection{Méthode des matrices de transfert}

On représente le circuit hydraulique comme une suite de composants élémentaires auxquels on cherche à associer un modèle de comportement indépendant du reste du circuit (réf. [4], [5], [13]).

En admettant que les perturbations des 2 variables pression-débit descriptives de l'écoulement sont faibles devant les grandeurs moyennes correspondantes, alors pour chaque pulsation, l'amplitude complexe des fluctuations à la sortie du composant s'exprime linéairement en fonction de l'amplitude des fluctuations d'entrée.

On peut démontrer les propriétés suivantes:

- La matrice de transfert d'un composant possédant un plan de symétrie orthogonal à son axe a un déterminant valant un et des coefficients diagonaux égaux.
- La matrice de transfert d'un élément à caractéristiques linéiques constantes satisfait à l'équation fonctionnelle :

$$
M(x+y)=M(x) \cdot M(y)
$$

dont la solution générale est de la forme :

$$
M(x)=\exp (x K)
$$

où $K$ est une matrice carrée d'ordre 2 vérifiant l'équation matricielle :

$$
\frac{\partial}{\partial x}\left[\begin{array}{l}
p \\
q
\end{array}\right]=K\left[\begin{array}{l}
p \\
q
\end{array}\right]
$$

Ce qui permet de calculer les coefficients de $K$ par identification du système différentiel décrivant l'écoulement.

La quantité $(p / \rho c)^{2}+(q / s)^{2}$ est invariante tout le long d'une conduite droite cylindrique.

Le principe d'assemblage des matrices associées aux différents composants d'un circuit est le suivant :

- Calcul de la matrice de transfert de 2 éléments associés en série (figure 14):

$$
\begin{gathered}
{\left[\begin{array}{l}
H_{1} \\
Q_{1}
\end{array}\right]=\left[\begin{array}{ll}
A_{11} & A_{12} \\
A_{21} & A_{22}
\end{array}\right]\left[\begin{array}{l}
H_{2}^{i} \\
Q_{2}^{i}
\end{array}\right]} \\
{\left[\begin{array}{l}
H_{2}^{i+1} \\
Q_{2}^{i+1}
\end{array}\right]=\left[\begin{array}{ll}
B_{11} & B_{12} \\
B_{21} & B_{22}
\end{array}\right]\left[\begin{array}{l}
H_{3} \\
Q_{3}
\end{array}\right]}
\end{gathered}
$$

Il y a équilibre des pressions au nœud 2 et conservation du débit:

$$
H_{2}^{i}=H_{2}^{i+1} \quad ; \quad Q_{2}^{i}=Q_{2}^{i+1}
$$

D'où :

$\left[\begin{array}{l}H_{1} \\ Q_{1}\end{array}\right]=\left[\begin{array}{ll}A_{11} & A_{12} \\ A_{21} & A_{22}\end{array}\right]\left[\begin{array}{ll}B_{11} & B_{12} \\ B_{21} & B_{22}\end{array}\right]\left[\begin{array}{l}H_{3} \\ Q_{3}\end{array}\right]$

La matrice $T$ des 2 éléments assemblés est égale au produit des matrices de chacun des éléments.

- Calcul de la matrice de transfert de 2 éléments associés en parallèle (figure 15):

$$
\begin{gathered}
{\left[\begin{array}{l}
H_{1}^{i} \\
Q_{1}^{i}
\end{array}\right]=\left[\begin{array}{ll}
A_{11} & A_{12} \\
A_{21} & A_{22}
\end{array}\right]\left[\begin{array}{l}
H_{2}^{i} \\
Q_{2}^{i}
\end{array}\right]} \\
{\left[\begin{array}{l}
H_{1}^{i+1} \\
Q_{1}^{i+1}
\end{array}\right]=\left[\begin{array}{ll}
B_{11} & B_{12} \\
B_{21} & B_{22}
\end{array}\right]\left[\begin{array}{l}
H_{2}^{i+1} \\
Q_{2}^{i+1}
\end{array}\right]}
\end{gathered}
$$

Il y a équilibre des pressions aux nœuds 1 et 2 et conservation du débit :

$$
H_{1}^{i}=H_{1}^{i+1}=H_{1} \quad ; \quad H_{2}^{i}=H_{2}^{i+1}=H_{2}
$$$$
Q_{1}=Q_{1}^{i}+Q_{1}^{i+1} \quad ; \quad Q_{2}=Q_{2}^{i}+Q_{2}^{i+1}
$$

D'où :

$$
\left[\begin{array}{l}
H_{1} \\
Q_{1}
\end{array}\right]=\left[\begin{array}{ll}
T_{11} & T_{12} \\
T_{21} & T_{22}
\end{array}\right]\left[\begin{array}{l}
H_{2} \\
Q_{2}
\end{array}\right]
$$

avec : 


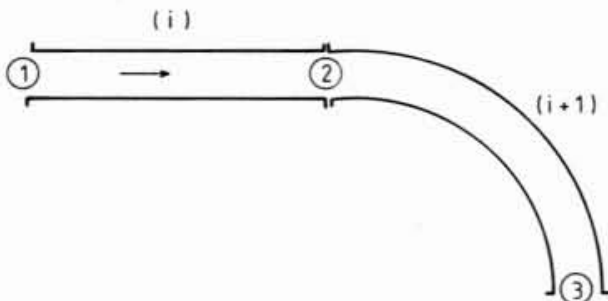

14.

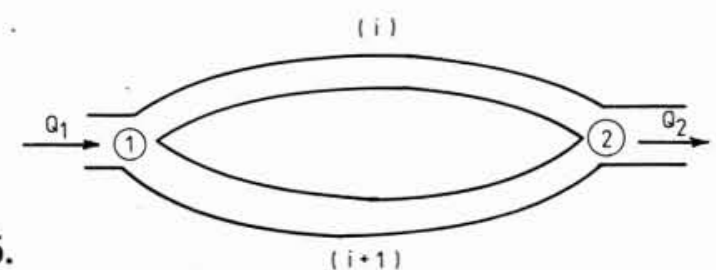

14. Association d'éléments en sêrie.

15. Association déléments en parallèle.

16. Cas 1 : caractéristiques du circuit.
$T_{11}=\frac{A_{11} B_{12}+A_{12} B_{11}}{A_{12}+B_{12}} \quad ; \quad T_{12}=\frac{A_{12} B_{12}}{A_{12}+B_{12}}$

$T_{21}=\frac{\left(A_{12} B_{12}\right)\left(A_{21}+B_{21}\right)-\left(A_{11}-B_{11}\right)\left(A_{22}-B_{22}\right)}{A_{12}+B_{12}}$

$T_{22}=\frac{A_{12} B_{22}+A_{22} B_{12}}{A_{12}+B_{12}}$

Le tableau des matrices de transfert associées aux principaux composants des circuits hydrauliques est donné en annexe. On distingue la matrice d'une conduite de section constante, celle d'une conduite de section variable, celle associée aux pertes de charge singulière, et les quelques modèles classiques utilisées pour les pompes. On mentionne également le cas de l'accumulateur.

\subsection{Interprétation des cas recensés}

Les quelques cas mentionnés dans l'introduction où l'on a observé le phénomène de résonance hydraulique ont fait l'objet d'une analyse par la méthode des matrices de transfert. Voici les principaux résultats obtenus sur chacun.

\section{Cas 1:}

Le modèle employé néglige l'ensemble des singularités de la ligne (coudes, piquages, vannes, bifurcations) et ne prend en compte finalement que les longueurs et les sections des tuyauteries considérées comme des éléments de tronçon droit. Ses caractéristiques sont représentées sur la figure 16. La célérité des ondes est supposée constante dans toute la ligne et prise égale à : $c=1130$ $\mathrm{m} / \mathrm{s}$.

Dans le $1^{\text {er }}$ cas, la pompe $\varnothing 2 \mathrm{PO}$ est à l'arrêt, et l'on impose une condition de fond plein $Q_{2}=0$ au nœud 2 .

On impose une condition de réflexion totale à la bâche $p_{4}=0$ au nœud 4 .

On écrit l'équilibre des pressions et la continuité au point de bifurcation, à savoir :

$$
\begin{aligned}
& p_{1}=p_{2}=p_{3} \\
& q_{1}+q_{2}=q_{3} \\
& \text { au nœud } 3
\end{aligned}
$$

En posant

$$
\alpha=\frac{\omega L}{c} \quad ; \quad \alpha_{1}=\frac{\omega L_{1}}{c} \quad ; \quad \alpha_{2}=\frac{\omega L_{2}}{c}
$$

et en écrivant les relations aux nœuds par la méthode des matrices de transfert, il vient:

$$
\left[\begin{array}{l}
P_{1} \\
Q_{1}
\end{array}\right]=\left[\begin{array}{cc}
\cos \alpha_{1} & -j \frac{\rho c}{S_{1}} \sin \alpha_{1} \\
-j \frac{S_{1}}{\rho c} \sin \alpha_{1} & \cos \alpha_{1}
\end{array}\right]\left[\begin{array}{l}
p_{1} \\
q_{1}
\end{array}\right]
$$$$
\left[\begin{array}{l}
P_{2} \\
Q_{2}
\end{array}\right]=\left[\begin{array}{cc}
\cos \alpha_{2} & -j \frac{\rho c}{S_{2}} \sin \alpha_{2} \\
-j \frac{s_{2}}{\rho c} \sin \alpha_{2} & \cos \alpha_{2}
\end{array}\right]\left[\begin{array}{l}
p_{2} \\
q_{2}
\end{array}\right]
$$

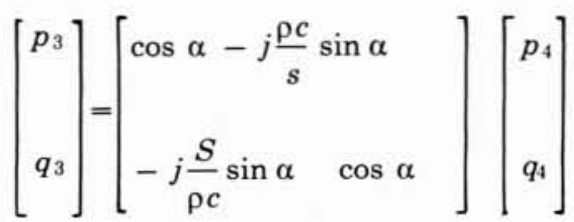




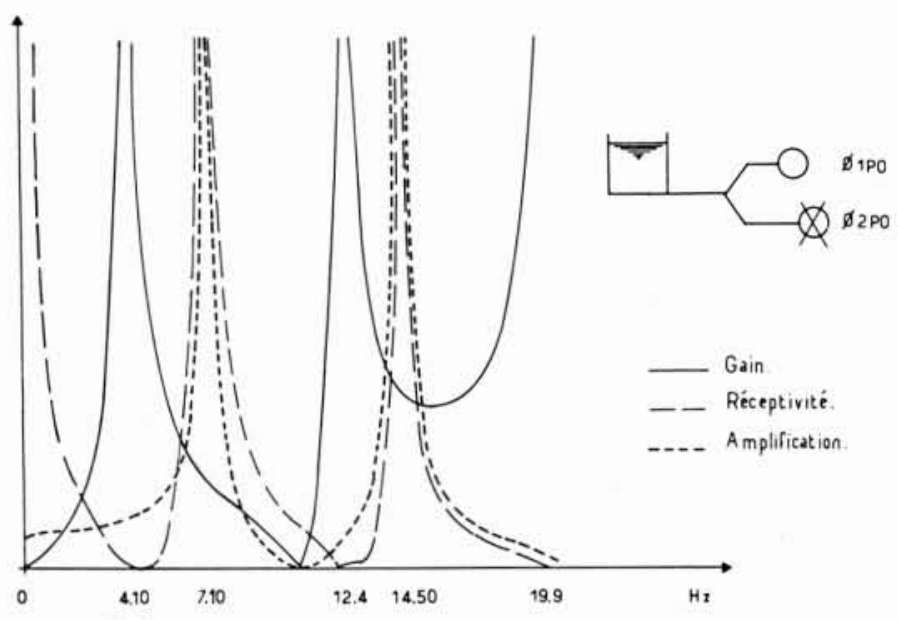

17. Cas 1: réponse du circuit avec la pompe $02 P O$ à l'arrêt.

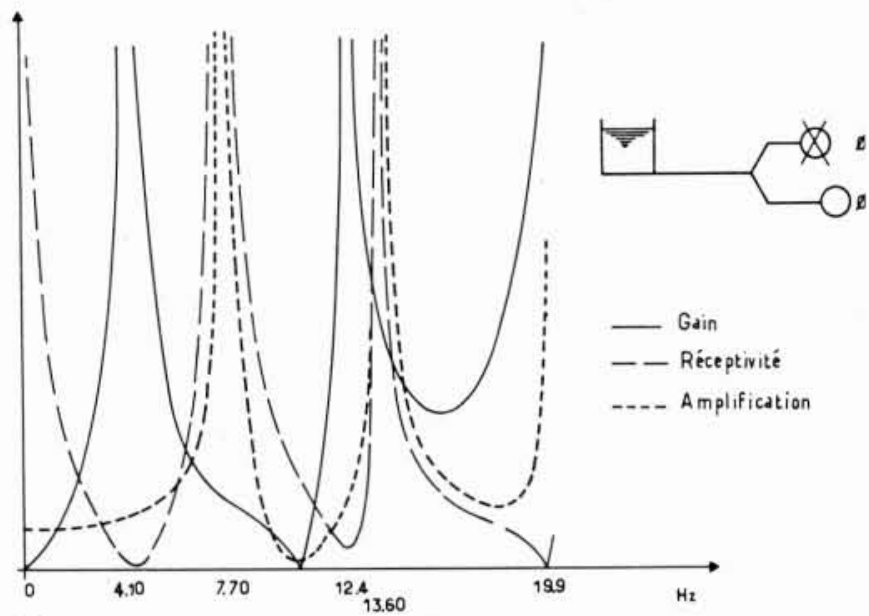

18. Cas 1: réponse du circuit avec la pompe Ø1PO à l'arrêt.
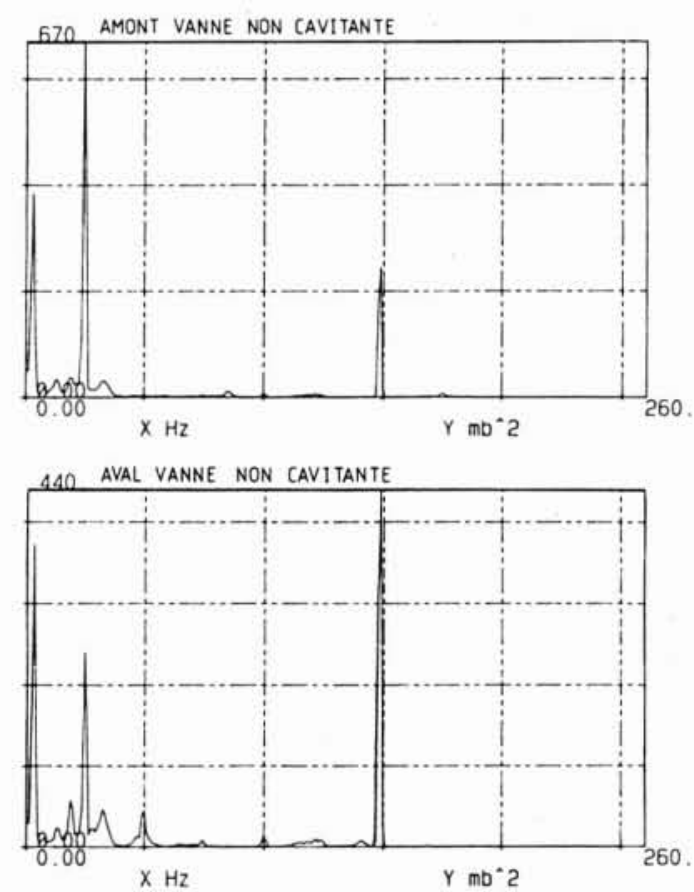

19. Autospectres des fluctuations de pression de la vanne non cavitante.
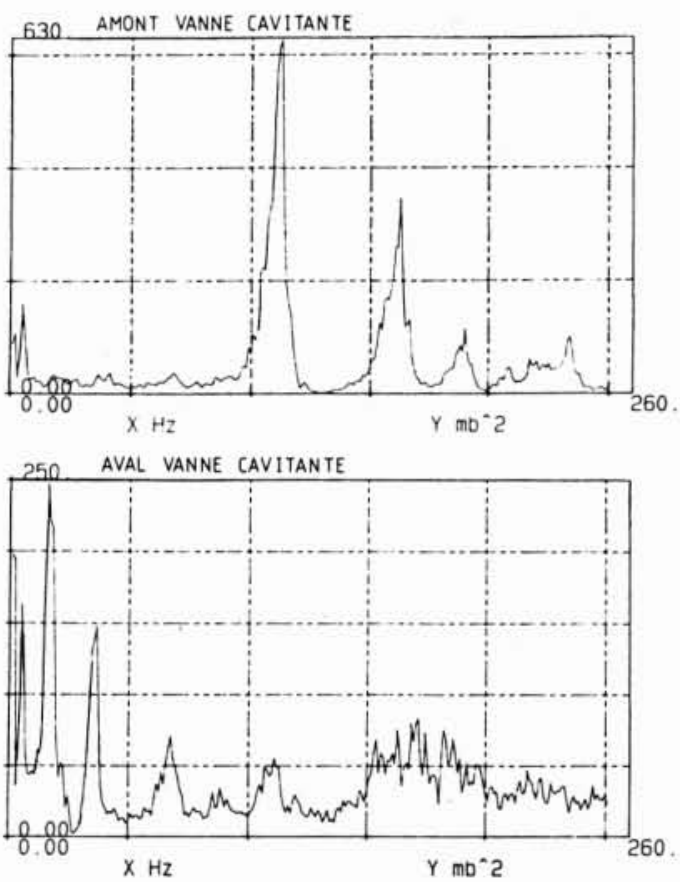

20. Autospectres des fluctuations de pression de la vanne cavitante.
En tenant compte des conditions aux limites et des conditions de raccordement, il vient :

$P_{1}=\left[\cos \alpha_{1}+\frac{S}{S_{1}} \frac{\sin \alpha_{1}}{\operatorname{tg} \alpha_{1}}-\frac{S_{2}}{S_{1}} \sin \alpha_{1} \operatorname{tg} \alpha_{2}\right] p_{1}$

$Q_{1}=\frac{j}{\rho c} \frac{1}{\cos \alpha_{2}}\left[S \frac{\cos \alpha_{1} \cos \alpha_{2}}{\operatorname{tg} \alpha}-S_{1} \sin \left(\alpha_{1}+\alpha_{2}\right)\right] p_{1}$

$P_{2}=\frac{1}{\cos \alpha_{2}} p_{1}$

Dès lors, on peut faire apparaître les quantités suivantes, appellées respectivement receptivité et gain :

$$
\frac{Q_{1}}{P_{1}}=-j \frac{S_{1}}{\rho c} \frac{S \frac{\cos \alpha_{1} \cos \alpha_{2}}{\operatorname{tg} \alpha}-S_{1} \sin \left(\alpha_{1}+\alpha_{2}\right)}{S \frac{\sin \alpha_{1} \cos \alpha_{2}}{\operatorname{tg} \alpha}+S_{1} \cos \left(\alpha_{1}+\alpha_{2}\right)}
$$$$
\frac{P_{2}}{Q_{1}}=-j \rho c \frac{1}{S \frac{\cos \alpha_{1} \cos \alpha_{2}}{\operatorname{tg} \alpha}-S_{1} \sin \left(\alpha_{1}+\alpha_{2}\right)}
$$

Et leur produit, qu'on appelle amplification :

$$
\frac{P_{2}}{P_{1}}=\frac{1}{\frac{S}{S_{1}} \frac{\sin \alpha_{1} \cos \alpha_{2}}{\operatorname{tg} \alpha}+\cos \left(\alpha_{1}+\alpha_{2}\right)}
$$


L'évolution de ces paramètres au cours d'un balayage en fréquence sur la gamme $(0-20 \mathrm{~Hz})$ avec une résolution de $0,01 \mathrm{~Hz}$ met en évidence 2 zones d'amplification correspondant aux 2 premières résonances du circuit centrées sur les fréquences $\omega_{r_{1}}=7,10 \mathrm{~Hz}$ et $\omega_{r_{2}}=14,50$ $\mathrm{Hz}$ (figure 17).

Dans le $2^{\mathrm{e}}$ cas, lorsqu'on intervertit les rôles de chacune des pompes, la pompe $\varnothing 1 \mathrm{PO}$ étant donc à l'arrêt, on observe encore 2 zones étroites d'amplification coïncidant avec les 2 premières résonances du circuit, et centrées sur les fréquences $\omega_{r_{1}}=7,70 \mathrm{~Hz}$ et $\omega_{r 2}=13,60$ $\mathrm{Hz}$ (figure 18 ).

Ces résultats appellent les remarques suivantes : si le phénomène d'amplification d'une branche sur l'autre est présent dans chaque cas, en revanche la dissymétrie géométrique de la ligne d'aspiration se retrouve au niveau des fréquences de résonance, qui ont glissé d'un cas à l'autre. Mais ce glissement est faible parce qu'il correspond à des résonances portant sur l'ensemble de la ligne d'aspiration qui comporte un tronçon commun aux 2 pompes d'une longueur beaucoup plus importante que celle des branches en parallèle. S'il semble douteux qu'un calcul introduisant les matrices de transfert singulières associées aux singularités du circuit puisse corriger ce glissement et rétablir la symétrie trouvée expérimentalement, en revanche une modélisation du système fluide-structure couplant les modes transverses de vibration de la ligne et la propagation en ondes planes est susceptible de restituer celle-ci, d'autant qu'il apparait que le $1^{\text {er }}$ mode de flexion de l'une des branches est extrêmement voisin de la $2^{\mathrm{e}}$ résonance hydraulique calculée qui de plus correspond pratiquement au $1^{\text {er }}$ harmonique fondamental.

Le couplage acoustique-mécanique doit être très sensible dans cette plage de fréquences.

\section{Cas 2:}

La méthode d'intensimétrie hydro-acoustique a permis d'évaluer les puissances directes et inverses propagées de part et d'autre de la vanne étudiée. En particulier elle a mis en évidence la dominance de la source associée à cette vanne dans le cas du régime fortement cavitant et sa non dominance hors cavitation. Elle a également permis d'estimer la célérité des ondes pour chacun des deux régimes : $1300 \mathrm{~m} / \mathrm{s}$ en amont et en aval de la vanne non cavitante, $1300 \mathrm{~m} / \mathrm{s}$ en amont de la vanne cavitante, $473 \mathrm{~m} / \mathrm{s}$ en aval.

Les raies qui apparaissent sur les spectres de puissances acoustiques sont essentiellement liées à la rotation de la pompe dans le cas non cavitant, auxquelles s'ajoutent des raies d'un niveau plus faible à $13,22,33$ et $85 \mathrm{~Hz}$ (figure 19) en amont de la vanne, et à 13, 20,33, 50 et $85 \mathrm{~Hz}$ en aval de la vanne (figure 19). Dans le cas cavitant en revanche, seule la raie à $25 \mathrm{~Hz}$ correspondant à la rotation est présente à la fois en amont et en aval. Les raies dominantes en amont sont en l'occurrence 45 , $70,115,125$ et $160 \mathrm{~Hz}$, et en aval : $16,36,50,70,85,110$ $\mathrm{Hz}$ (figure 20). On voit que ces fréquences sont très différentes de part et d'autre de la vanne.

La vanne est située sur une ligne de diamètre $\varnothing 100$ raccordée par un convergent à une ligne de diamètre $\varnothing 200$ situé à $11,5 \mathrm{~m}$ en amont et par un divergent à une ligne de diamètre $\varnothing 200$ situé à $12 \mathrm{~m}$ en aval. Ces éléments réflecteurs sont à l'origine de résonances locales avec la vanne, comme le confirme l'analyse du rapport des ondes inverse et directe, dont le module fournit le coefficient de réflexion et dont l'évolution linéaire de la phase en fonction de la fréquence permet de localiser le lieu de réflexion. Les fréquences de résonance en demi-longueur d'onde $(f=(k c) /(2 L)$; $k=1,2,3, \ldots)$ valent à l'amont 57,114 et $171 \mathrm{~Hz}$ (à comparer à 45,115 et $160 \mathrm{~Hz}$ sur les spectres), à l'aval $20,39,59 \mathrm{~Hz}$ (à comparer à 16,36 et $50 \mathrm{~Hz}$ ).

En réalité, c'est l'ensemble du circuit qui participe à la résonance hydraulique, même si certaines parties sont beaucoup plus sensibles que d'autres à certaines fréquences. De plus les coefficients de réflexion des singularités du circuit peuvent varier avec la fréquence, ce qui complique l'analyse en demi-longueur d'onde pour laquelle on choisissait forfaitairement un coefficient de réflexion égal à l'unité. La mise en œuvre de la méthode des matrices de transfert pour l'ensemble du circuit permet d'obtenir les fréquences pour lesquelles l'énergie acoustique émise par la vanne présente une amplification maximum (figures 21, 22, page suiv.). Dans le cas non cavitant, on observe en entrée et en sortie de vanne sensiblement les mêmes pics aux fréquences $22,32,68$, 80 , une raie très importante à $86 \mathrm{~Hz}$, de l'énergie répartie à 135 et $145 \mathrm{~Hz}$, à nouveau des pics au-delà de $180 \mathrm{~Hz}$. Par rapport aux résultats expérimentaux, on retrouve bien 22,33 et $85 \mathrm{~Hz}$ dont on peut affirmer qu'il s'agit bien de fréquences propres de circuit. Il est probable également que l'énergie disponible, juste en-dessous de la fréquence de passage des aubes $(150 \mathrm{~Hz})$ contribue à amplifier cette raie, d'autant que l'on remarque sur certains autospectres de pression la présence d'un pic au flanc gauche du $150 \mathrm{~Hz}$. Dans le cas cavitant (qui au plan de la méthode mise en œuvre ne se distingue du précédent que par la prise en compte du changement de célérité des ondes au passage de la vanne), on relève en entrée les raies énergétiques suivantes : $13,25,40,65,74$, $85,122,140,162 \mathrm{~Hz}$ et quelques fréquences encore au-delà. En sortie on retrouve exactement ces mêmes fréquences, ce qui semble être en contradiction avec le résultat expérimental. En fait, c'est que la méthode de calcul employée ne partage pas le circuit en deux parties "étanches" au niveau de la vanne, comme c'est le cas en réalité, de sorte que les fréquences d'entrée, filtrées en sortie (et réciproquement), se retrouvent de fait de part et d'autre dans le calcul. On propose alors de comparer 40,122 et $162 \mathrm{~Hz}$ à $45,115-125$ et $160 \mathrm{~Hz}$ pour l'amont; 13,25 et 65 à 16, 36 et 50 pour l'aval mais il faut bien admettre que la représentation adoptée pour la vanne cavitante s'avère insuffisante pour rendre compte des phénomènes mesurés.

\section{Cas 3:}

Dans le troisième exemple mentionné en introduction, l'analyse théorique montre que les fréquences de résonance hydraulique dépendent de l'ensemble des éléments du circuit et que l'effet capacitif dû aux déformations du corps de pompe est un terme qu'il importe de prendre en compte. Les articles des références [1] et [2] fournissent les principaux éléments de l'étude. 


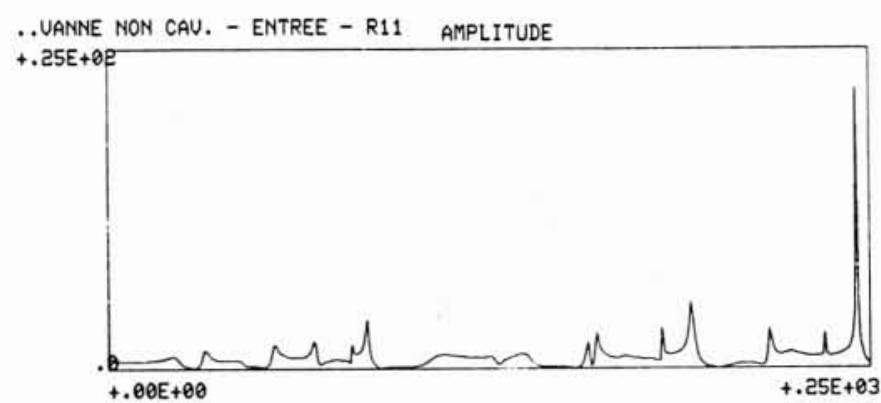

. UANNE NON CAU. - SORTIE - R11 AMPLITUDE

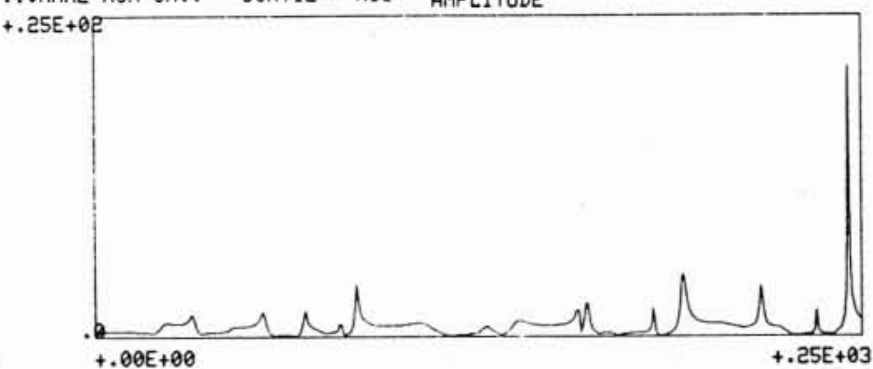

. UANNE NON CAU. - ENTREE - R12 AMPLITUDE
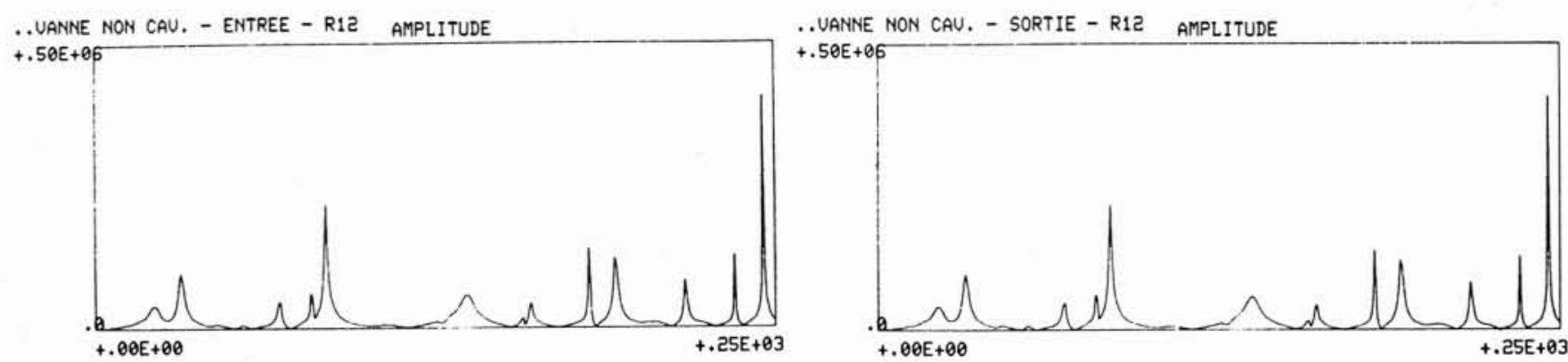

21 . Vanne non cavitante: résultats théoriques.
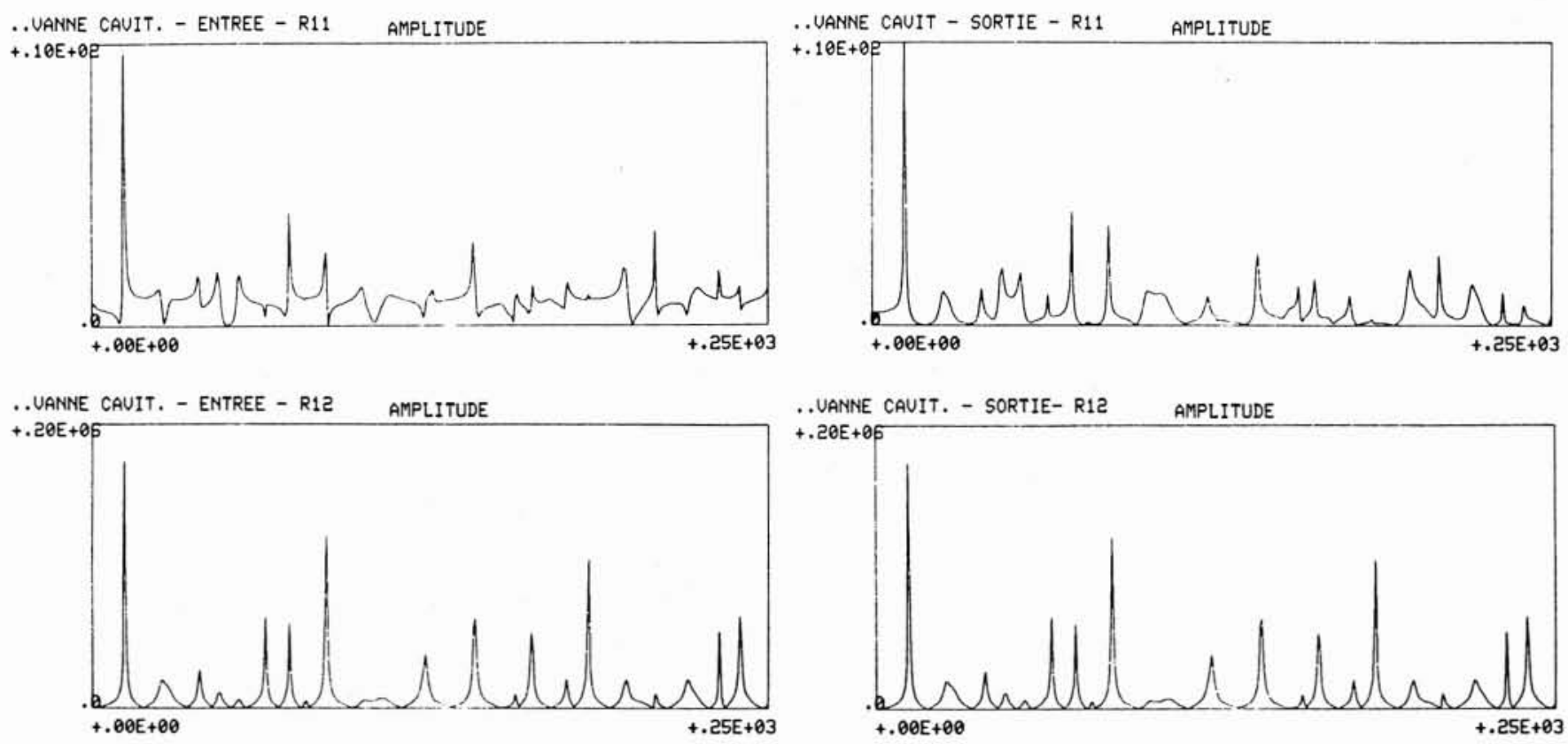

22. Vanne cavitante : résultats théoriques.

On retiendra notamment que plus le terme capacitif introduit dans les coefficients de la matrice de transfert de la pompe est important, plus les fréquences naturelles du circuit diminuent et plus des écarts importants apparaissent entre l'aspiration et le refoulement de la pompe. Si le calcul permet de prédire correctement les 2 premières fréquences naturelles, en revanche un glissement apparaît pour les fréquences plus élevées. 


\section{Conclusion}

La méthode des matrices de transfert conduit à des résultats convenables pour l'étude des interactions machine-circuit dans le domaine des basses fréquences. Des modèles théoriques de matrices de transfert sont disponibles pour les éléments hydrauliques simples mais les résultats expérimentaux restent indispensables pour caractériser les éléments complexes (vannes, pompes...). Pour ces éléments complexes la validité de la représentation par une matrice de transfert et un vecteur d'excitation n'est pas certaine car il est possible que les fluctuations qui s'établissent et qui dépendent du circuit aient une action sur la source. L'analyse de la linéarité du comportement des éléments complexes peut être envisagée en utilisant des techniques récentes du traitement du signal (transformée de Hilbert par exemple).

\section{Bibliographie}

[1] Desmet B. - Pressure Fluctuations Analysis at the Inlet of a Small Centrifugal Pump, IAHR Work Group on the Behaviour of Hydraulic Machinery Under Steady Oscilla. tory Conditions, 2nd Meeting, Mexico, 18-20 Septembre $1986,10 / 1-10 / 12$.

[2] Desmet B., Barrand J.P. - Analyse des fluctuations de pression à l'aspiration et au refoulement d'une pompe centrifuge, IAHR, $13^{e}$ Symposium, Montréal (CAN.), 2-5 Septembre 1986, Vol. 1, 18/1-18/14.

[3] JAEger C. - Fluid Transients in Hydro-Electric Engineering Practice, Blackie, 1977.

[4] Wilie E.B., Streeter V.L. - Fluid Transients, Mc Graw-Hill, 1978.

[5] Chaudhry M.H. - Applied Hydraulic Transients, Van Nostrand Reinhold Compagny, 1979.

[6] STECKI J.S., Davis D.C. - Fluid Transmission lines distributed parameter models. Part $1:$ a review of the state of the art. Proceedings of the Institution of Mechanical Engineers, Vol. 200, $\mathrm{n}^{\circ}$ A4, 1986, 215-228.

[7] FANelli M. - Nota sulla formula della celerità per onde di molto vario in condotte deformabili, non publié, 1978.
[8] Cantrell R.H., HaRT R.W. - Interaction between Sound and Flow in Acoustic Cavities: Mass, Momentum and Energy Considerations, Journal of the Acoustical Society of America, 16 (4), Avril 1964, 697-706.

[9] Morfey C.L. - Acoustic Energy in Non Uniform Flows, Journal of Sound and Vibrations, 14 (2), 1971, 101-109.

[10] Mundro D.H., INGard K.U. - On Acoustic Intensity Measurements in the Presence of Mean Flow, Journal of the Acoustical Society of America, 65 (6), Juin 1979, 1402-1406.

[11] JACOBSEN F. - Measurement of Sound Intensity in the Presence of Air Flow, $2^{e}$ Congrès International sur l'Intensimétrie Acoustique, CETIM, SENLIS (France), 23-26 Septembre 1985, 193-200.

[12] Badie-Cassagnet A., Bockoff, Lambret J.M. - Application de l'intensimétrie acoustique à l'identification des sources de pulsation de pression dans des circuits, Journée d'Etude "Hydraulique et Bruit", SHF - GALF, Paris, 15 décembre 1981, 261-268.

[13] Dordan J.J., MarchetTI M. - Matrices de transfert de systèmes hydrauliques. Etude théorique et expérimentale, La Recherche Aérospatiale, n 1974-1, Janv. Fév., 23-35.

$\begin{array}{lll} & \text { Adresses des auteurs } \\ \text { Monsieur B. Desmet } & \text { Monsieur F. Tephany } & \text { Monsieur J.L. Trolle } \\ \text { ENSAM } & \text { EDF-DER } & \text { EDF-DER } \\ \text { 8, bld Louis XIV } & \text { 6, quai Watier } & \text { 25, allée Privée } \\ \text { 59046 Lille Cedex } & 78400 \text { Chatou } & 93206 \text { Saint-Denis } \\ \text { Tél. : } 20.53 .11 .00 & \text { Tél. : 30.71.72.44 } & \text { Tél.: } 48.29 .90 .00\end{array}$




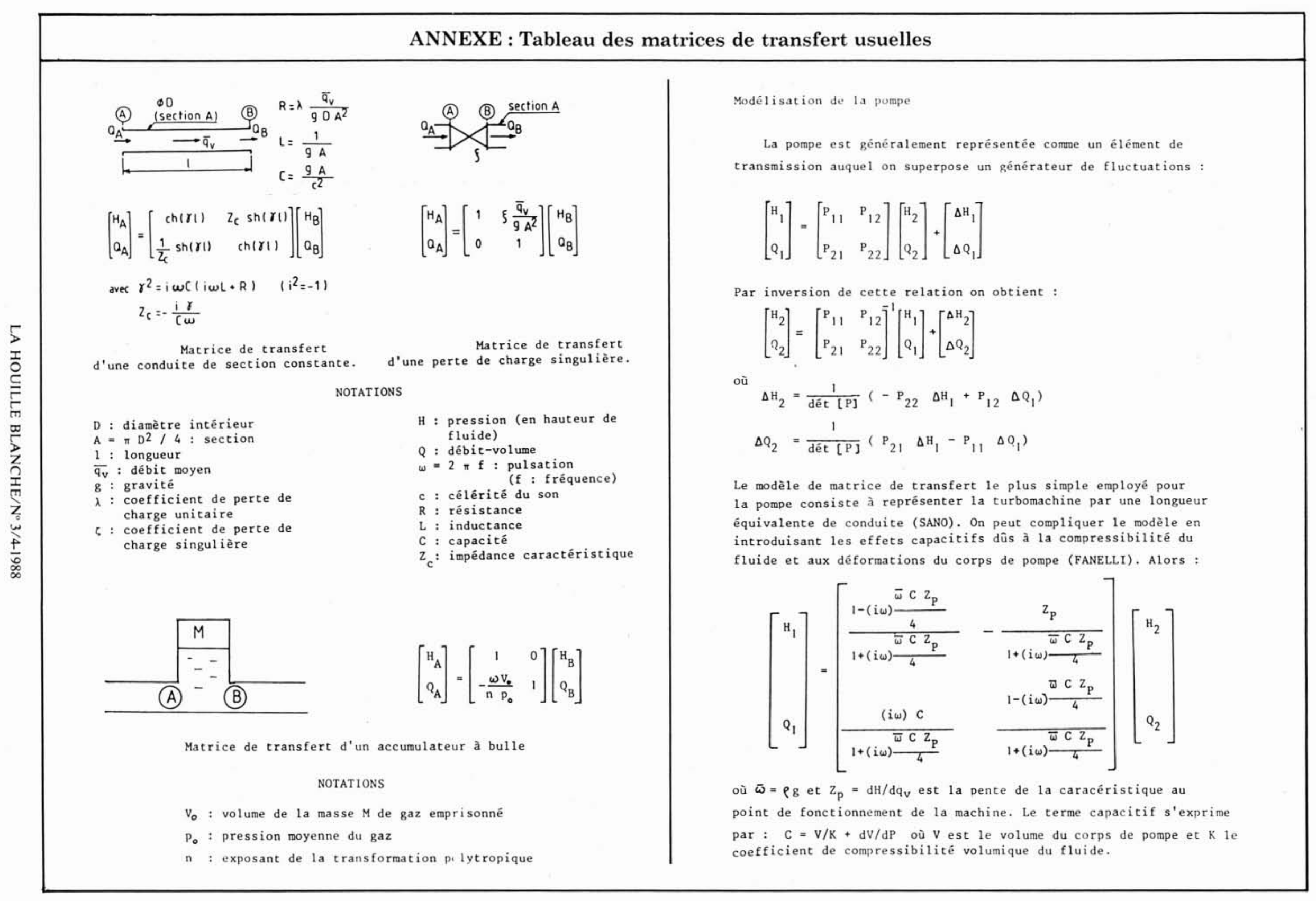


M. le Président. - Merci M. DESMET pour cet intéressant exposé. J'ai la question suivante à vous poser : dans tout circuit hydraulique, il y a des coudes. Quel est votre avis sur la validité de la théorie des ondes planes dans ces zones coudées ?

M. DESMET. - Question intéressante à laquelle il est difficile de répondre. Dans ce cas le champ des vitesses moyennes n'est plus du tout unidimensionnel.

Le problème est de savoir si pour les fluctuations ce caractère fortement tridimensionnel s'observe également... On ne dispose pas pour l'instant de mesures qui permettent de répondre ni dans un sens ni dans l'autre.

Mais si l'on introduit une singularité faible ou même une singularité relativement importante, telle qu'une vanne - et les résultats donnés par les essais réalisés sur la boucle d'EDF le confirment - on a une matrice de transfert pour le tronçon représenté qui diffère très peu de la matrice de transfert pour une conduite rectiligne. Il semble que l'influence de cette singularité, au moins dans le domaine des basses fréquences, reste relativement peu importante.

M. FABRI. - Je ne suis pas hydraulicien, je suis mécanicien des fluides; j'aurais aimé répondre à la question de M. CAILLOT de façon un peu différente.

Il est certain que dans le coude proprement dit, l'écoulement n'est pas unidimensionnel. Mais si l'on remarque que l'étendue longitudinale d'un coude est petite devant celle des tronçons rectilignes du circuit, à faible distance en amont et en aval du coude l'écoulement redevient quasi-unidimensionnel, et la théorie des ondes planes reste valable avec l'hypothèse d'un coude représenté par une discontinuité des paramètres hy. drauliques.

M. DESMET. - C'est ce que confirment les résultats expérimentaux.

M. ORDONNEAU. - Je n'ai pas fait d'expériences sur les systèmes hydrauliques, mais j'aurais voulu connaitre les types de débit-mètres que vous employez pour déterminer les matrices de transfert.

M. DESMET. - En général il n'y a pas de mesures de débit instantané réalisées. On fait des mesures de pression à l'amont et à l'aval de l'élément à étudier, et à partir de ces mesures on est capable de reconstituer la loi de pression, et en utilisant un modèle undimensionnel on peut revenir aux fluctuations de vitesse : c'est en général la technique adoptée.

Il en va de même pour les mesures de vitesse de propagation. On considère que la demi-somme des fonctions de transfert correspond à une fonction réelle qui doit être une sinusoïde; et la période de cette sinusoïde dépend de la vitesse de propaga. tion, ce qui fait qu'on peut remonter à la vitesse de propagation.
M. LEFEBVRE. - Etes-vous pleinement satisfait de la boucle Sésame? Quelles seraient les améliorations possibles?

M. DESMET. - Pour l'instant, on n'a pas trouvé de défauts qui la remettent en cause; mais on notera qu'on est en milieu fortement réverbérant, ce qui peut pour des mesures d'énergie des fluctuations poser certains problèmes.

Dans certains cas, il serait intéressant de réduire le taux d'ondes stationnaires par utilisation de terminaisons anéchoïques, pour éviter qu'il y ait trop de réverbération. Si l'on a un coefficient de réflexion égal à 1 , on peut avoir des niveaux de fluctuations importants, entretenus avec une énergie quasiment nulle.

M. TOURRET. - Quelle modélisation peut-on faire d'une pompe centrifuge dans le système de matrices de transfert? Quelles sont les conditions d'extrémités que vous avez prises pour les deux pompes centrifuges?

M. DESMET. - Dans le modèle, les pompes n'interviennent pas parce qu'elles sont en dehors du système étudié. La pompe n'est pas représentée dans le modèle.

\section{TOURRET. - Et si vous deviez la représenter ?}

M. DESMET. - Dans la matrice de transfert d'une pompe, une chose intervient déjà : c'est la pente de la caractéristique de la pompe au point de fonctionnement. Avec des modèles un peu évolués on peut faire intervenir les effets capacitifs de la pompe terme $\partial V \partial \partial$ ainsi que le terme en $\partial V \partial Q$. Mais ce qui manque encore, ce sont les confirmations expérimentales de ces modèles.

On pourrait aussi envisager de représenter la pompe par des matrices de transfert élémentaires, avoir un modèle pour représenter la zone d'aspiration, un autre modèle pour représenter la roue, un autre modèle encore pour représenter le diffuseur, et progressivement de proche en proche, on représenterait ainsi l'ensemble de la machine.

M. Tourret. - M. Bernard a fait au CETIM des expériences sur les coefficients de réflexion des coudes et de convergents ou divergents permettant des raccordements de sections.

Il apparaît qu'un coude de très grand rayon possède un coefficient de réflexion beaucoup plus faible dans le domaine " moyennes et basses fréquences" que les valeurs obtenues pour un rétrécissement de section extrêmement progressif pour lequel la réflexion est très importante. D'ailleurs, entre un rétrécissement brusque et un rétrécissement progressif, la différence est pratiquement nulle dans les basses fréquences.

M. le Président. - Merci Messieurs. 